\title{
Stochastic Noise Removal on Partial Discharge Measurement for Transformer Insulation Diagnosis
}

\author{
Jeffery C. Chan, Student Member, IEEE, Hui Ma, Member, IEEE, Tapan K. Saha, Senior Member, IEEE, Chandima \\ Ekanayake, Member, IEEE \\ The University of Queensland \\ Brisbane, Australia \\ j.chan@uqconnect.edu.au, huima@itee.uq.edu.au, saha@itee.uq.edu.au,chandima@itee.uq.edu.au
}

\begin{abstract}
Measurement of partial discharge (PD) paves a way for transformer insulation diagnosis. However, noise always interferes with PD signals and can jeopardize the diagnostic reliability. Therefore, it is necessary to adopt signal processing techniques to remove noise from collected signals. Among various types of noise, stochastic noise is considerably difficult to remove due to its similarity with PD signals. This paper proposes an effective method, which adopts fractal dimension and entropy analyses to remove stochastic noise. To verify the proposed method, PD measurements have been performed on a number of experimental models and a substation transformer. Results prove that PD signals can be extracted while the noise can be eliminated from collected noise-corrupted signals by using the proposed method. A comparison with a wavelet transform-based noise removal method has also been made in the paper.
\end{abstract}

Index Terms-De-noising, entropy, fractal dimension, partial discharge (PD), transformer.

\section{INTRODUCTION}

Transformer is one of the most crucial assets in electricity networks and the transformer's health conditions highly depend on its insulation system. Partial discharge (PD) measurement is an effective method for evaluating the insulation system [1], [2]. However, PD signals collected from PD measurements in substation environment are always overwhelmed by a variety of interference and noise. This can pose significant difficulties in recognizing $P D$ patterns generated from corresponding insulation defects in a transformer and making explicit diagnosis on its insulation system.

There are different types of noise that can exist during PD measurements, such as white noise, periodic impulsive and sinusoidal noise, and stochastic noise [3]-[6]. Among the above types of noise, stochastic noise is considerably difficult to remove and limited research works have been reported in the literature. In [7], neural network is used to remove stochastic noise. However, this method requires a training

This work was supported by the Australia Research Council (ARC) on Linkage Grant. process to create a database for classifying PD pulses generated by different PD sources. For those situations that collected PD signals do not belong to the database can induce misclassifications. The major difficulty on removing stochastic noise is due to its similarity in frequency characteristics with PD signals [6]. Therefore, conventional methods such as notch filtering and gating method are not able to provide an effective filtering for stochastic noise.

Recently, wavelet transform attains a high attention on removing noise from collected signals for PD measurements [3]-[6]. The principle of wavelet transform is based on signal decomposition through selected wavelet functions. PD signals can be separated from noise in the decomposed signals with different frequency scales. By applying thresholds to the decomposed signals and performing signal reconstruction, PD signals can be retrieved while noise can be removed. However, frequency scales of stochastic noise can be the same as those of PD signals. This implies that PD signals may not be distinguished from stochastic noise using wavelet transform. Since no research works address this issue, this paper (Section V) examines the capability on using wavelet transform for removing stochastic noise.

This paper proposes a hybrid of fractal dimension and entropy analyses for effectively distinguishing and extracting PD signals from collected signals corrupted by stochastic noise. Fractal dimension is a method to describe complex shapes, such as coastlines and mountains, by using fractal sets [9], while entropy is a measure of uncertainty in a signal. In the proposed method, fractal dimension is used to separate PD signals from the noise, which locates in distinctive power cycles from the PD signals. Meanwhile, entropy is calculated to distinguish and extract PD signals from the noise, which distributes over the power cycles with PD signals.

The paper is organized as follows. Section II presents experimental set-up of PD measurements. Section III provides a brief introduction on fractal dimension and entropy. Section IV describes the proposed method on stochastic noise removal and PD signal extraction from raw signals (i.e. noise-corrupted signals) collected from PD measurements. Section V presents 
results by applying the proposed method to measured signals obtained from both experimental models and a substation transformer. A comparison with a wavelet transform-based noise removal method is also provided. Section VI concludes the paper.

\section{EXPERIMENTAL SET-UP}

The PD measurement set-up used in this paper is based on IEC 60270 standard (Fig. 1) [10]. In the set-up, V is a voltage source, $\mathrm{C}_{\mathrm{a}}$ is a test object (i.e. a PD experimental model or a transformer), $\mathrm{C}_{\mathrm{k}}$ is a coupling capacitor, $\mathrm{Z}$ is measuring impedance, and $\mathrm{M}$ is a data acquisition and processing system. PD experimental models simulate PD sources using different configurations (Fig. 2). The model shown in Fig. 2a simulates discharge with flat cavity by putting four pressboards (diameter $=40 \mathrm{~mm}$ ) together in transformer oil with a cavity (diameter $=15 \mathrm{~mm}$ ) at the center. The model shown in Fig. $2 b$ simulates corona with $50 \mathrm{~mm}$ distance between a needle and grounding. The model shown in Fig. 2c placed in air simulates surface discharge by using one pressboard putting between the needle and grounding. These experimental models were used to generate both single and multiple PD sources.

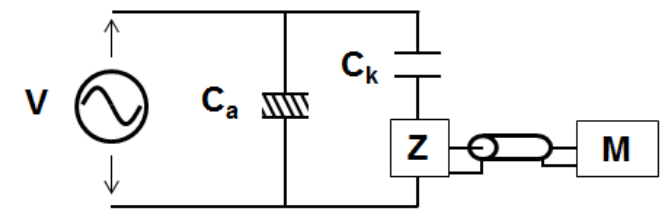

Figure 1. PD measurement set-up.

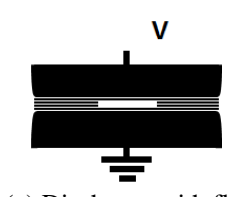

(a) Discharge with flat cavity

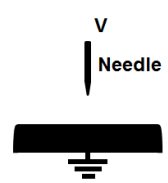

(b) Corona

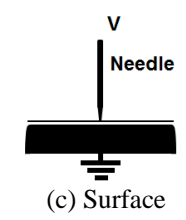

discharge

Figure 2. PD experimental models.

\section{INTRODUCTION ON FRACTAL DIMENSION AND ENTROPY}

\section{A. Fractal Dimension}

The concept of fractal dimension refers to changes of a pattern's details with respect to the scales used for measuring this pattern. A number of methods including box-counting, variance, and spectral methods are used for calculating fractal dimension [11]. This paper adopts box-counting for its simplicity and efficiency [12]. Fractal dimension (FD) of a signal in Euclidean space is defined as [13]:

$$
F D=\lim _{\varepsilon \rightarrow 0} \frac{\log N(\varepsilon)}{\log (1 / \varepsilon)}
$$

where $N(\varepsilon)$ is the least number of boxes with side length $\varepsilon$ to cover the signal.

A PD source caused by a particular insulation defect can exhibit a unique PD pattern. Due to its ability of representing various patterns and describing complex shapes, fractal dimension has been used for feature extraction and recognition

of PD sources [13], [14]. This paper makes use of fractal dimension to remove and quantify severity of noise.

\section{B. Entropy}

When PD signals and noise merge together, fractal dimension alone may not be able to recover PD signals and thus entropy is used. Entropy is a measure of disorder in a random variable. A larger value of entropy relates to more chaotic data [15]. For a signal $X=\left[x_{1}, x_{2}, \ldots, x_{n}\right]$, its entropy, $H(X)$, is defined as:

$$
H(X)=-\sum_{i=1}^{n} p\left(x_{i}\right) \log _{2} p\left(x_{i}\right)
$$

where $p\left(x_{i}\right)$ is the probability mass function of $x_{i}$.

This paper adopts entropy to find how abundant information is in each box of fractal dimension. This can help to differentiate PD signals from noise. Next section will provide more details on the application of fractal dimension and entropy for stochastic noise removal on PD measurements.

\section{Proposed Method on Stochastic Noise Removal}

Fig. 3 depicts the flowchart of the proposed stochastic noise removal method for PD measurements. Firstly, a collected one-dimensional (1D) noise-corrupted signal consisting of both PD signal and stochastic noise is transformed into a two-dimensional (2D) signal. In this transformation, the original signal in a power cycle is converted to 512 x 512 pixels for accurately representing the signal while still maintaining reasonable processing time in subsequent fractal dimension and entropy calculations. Then, fractal dimension is calculated on the transformed 2D signal in each power cycle with different side lengths of boxs, $2^{x}$, where $x=[1,2, \ldots, n]$. If severe noise presents in a particular power cycle, the value of fractal dimension in this power cycle is different from that of others. Thus, it is able to indicate the present of noise. Subsequently, the noise can be removed by discarding the signal in the power cycle with noise and selecting the signals in other cycles.

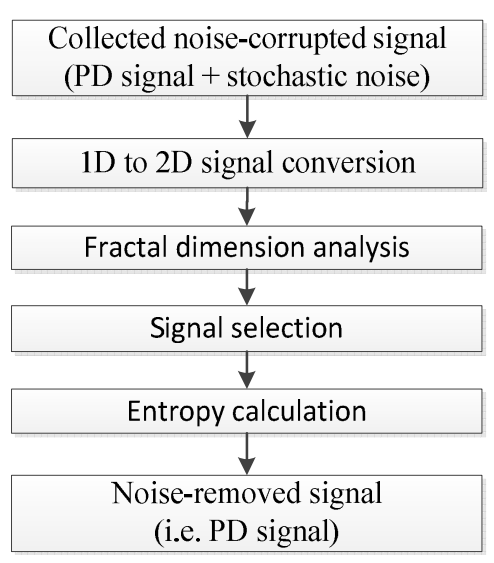

Figure 3. Proposed stochastic noise removal method.

Since noise may locate in every or most of power cycles with similar fractal dimension values, using fractal dimension 
alone may not be able to completely distinguish and separate PD signals from noise. Moreover, even at the situations that fractal dimension can distinguish noise appearing at most of power cycles; it is still not wise to discard all signals located in those power cycles. Therefore, entropy is calculated in each box within each power cycle.

For a signal generated by stochastic noise, if it locates in a particular box in a power cycle, it is unlikely present at the same box in other power cycles when compared with PD signals. Based on this assumption, entropy value of the noise in each box is not as high as that of PD signals. After adding entropy values of the same box for every power cycle together and using color to represent the sum (the highest to lowest values are represented by a colormap ranging from red, orange, yellow, cyan, to blue), PD signals (i.e. noise-removed signals) can be identified and subsequently extracted from noisecorrupted signals.

\section{RESUlTS AND DisCUSSIONS}

To evaluate the proposed method on stochastic noise removal, PD signals were collected from PD measurements on the experimental models (Fig. 2). PD signals were also collected from a PD measurement on a 5 MVA power transformer for further evaluation of the proposed method. A comparison with a wavelet transform-based noise removal method has also been made in this section to justify the effectiveness of the proposed method.

\section{A. Noise Removal Evaluation on PD Experimental Models}

Fig. 4 presents noise removal results on a PD measurement performed for the PD experimental model of discharge with flat cavity (i.e. Fig. 2a). From Fig. 4a (original PD measurement signal), it can be observed that the PD signal locates periodically in most of power cycles. Severe stochastic noise occurs at the $1^{\text {st }}$ and $2^{\text {nd }}$ power cycles where relatively insignificant noise can be found at the $3^{\text {rd }}$ power cycle. The stochastic noise was generated by occasional discharges from the transformer supplying voltage to the PD measurement system and the coupling capacitor in the measurement circuit (Fig. 1). This is because both the transformer and coupling capacitor have been used for more than 20 years and cannot be regarded as discharge free.

Fig. $4 \mathrm{~b}$ presents normalized results of fractal dimension in each power cycle with different side lengths of boxes. The numbers in Fig. 4b represent the power cycles' numbers. From the figure, it can be seen that fractal dimensions from the $1^{\text {st }}$ to the $3^{\text {rd }}$ cycles are separated from those of other cycles in most of side lengths. The fractal dimension of the $3^{\text {rd }}$ cycle containing minor noise is close to those from the $4^{\text {th }}$ to the $10^{\text {th }}$ cycles. By contrast, fractal dimensions of the $1^{\text {st }}$ and $2^{\text {nd }}$ cycles containing severe noise are relatively far from those from the $4^{\text {th }}$ to the $10^{\text {th }}$ cycles. These results can be used for distinguishing and separating PD signals from noise.

By rejecting signals in the first three power cycles and combining the signals in the remaining cycles into one cycle, phase-resolved partial discharge (PRPD) pattern can be obtained (Fig. 4d). PRPD patterns reveal signals distribution with respect to phase angles of applied test voltages [8]. Fig. $4 \mathrm{c}$ is the original PRPD without applying the proposed noise removal method. It can be seen that PD pattern in the original PRPD is contaminated by stochastic noise. This demonstrates that the original PRPD cannot fully represent the PD pattern caused by the corresponding insulation defect (i.e. discharge with flat cavity) and thus may not be suitable for transformer insulation diagnosis.

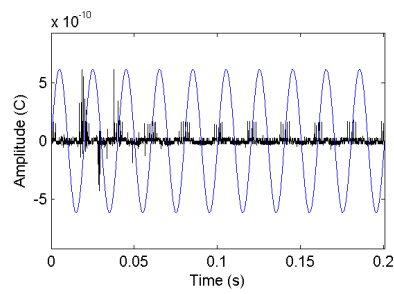

(a) Original signal

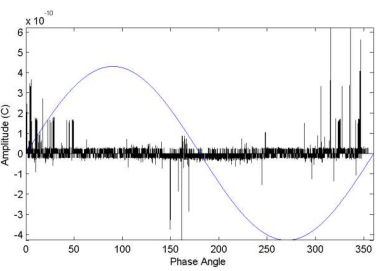

(c) Original phase-resolved partial discharge (PRPD)

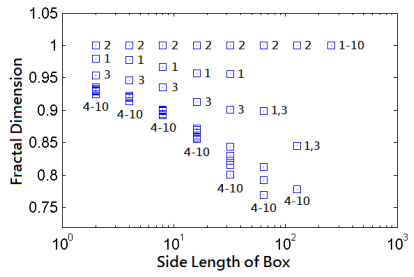

(b) Fractal dimension

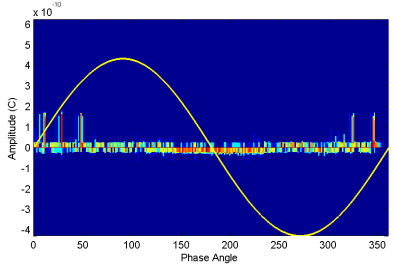

(d) Noise-removed phase-resolved partial discharge (PRPD)
Figure 4. Noise removal results of discharge with flat cavity. (Note: The numbers in Fig. 4b represent power cycles' numbers)

The results in Fig. 4 prove that fractal dimension used by the proposed method is capable of identifying and removing stochastic noise. It can also indicate severity of noise by comparing its value of signal in each power cycle. For those signals that noise is located in every power cycle and the signals themselves have similar values of fractal dimensions, entropy is employed to further eliminate the noise. The results of applying entropy for removing noise are presented in Fig. 5.

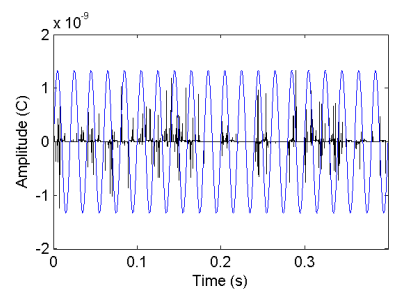

(a) Original signal

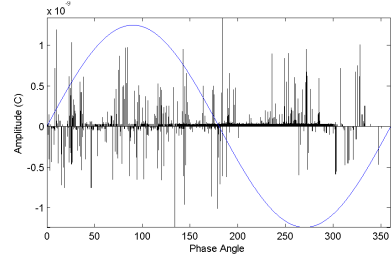

(c) Original phase-resolved partial discharge (PRPD)

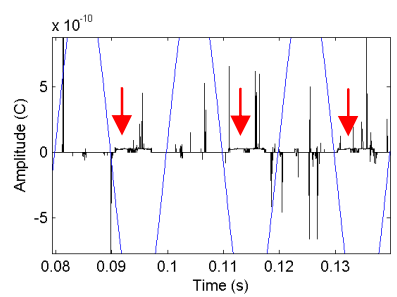

(b) Original signal (magnified)

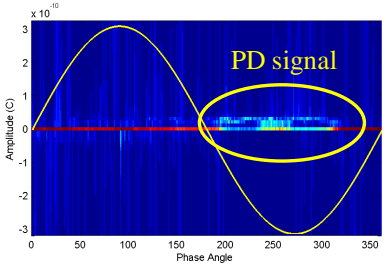

(d) Noise-removed phase-resolved partial discharge (PRPD, magnified)
Figure 5. Noise removal results of corona.

(Note: In Fig. $5 \mathrm{~d}$ the pulses in blue color are stochastic noise) 
In Fig. 5, the PD signal is caused by corona (Fig. 2b). During the measurement, the applied voltage was below $5 \mathrm{kV}$ and distance between the needle and grounding was kept at $50 \mathrm{~mm}$. The purpose of such arrangement is to generate corona with small amplitudes (when compared with the noise) for evaluating performance of the proposed noise removal method on revealing small-amplitude PD signals. From the original signal and its magnification (Fig. 5a and Fig. 5b), noise can be observed in most of power cycles. In contrast, the discharge signals (indicated by red arrows, Fig. 5b) are hardly identified due to their relatively small amplitudes. Though some noise can be identified in some power cycles and removed with fractal dimension calculation, other noise still presents in the remaining power cycles (since the signals in these power cycles have similar fractal dimension values). Through calculating entropy in each box of fractal dimension in each cycle and then combining all entropy values into a single cycle, noise-removed PRPD is obtained (Fig. 5d). PD signal is clearly identified (the pulses represented by blue color are stochastic noise). However, in the original PRPD without applying the proposed noise removal method, PD signal is submerged in noise (Fig. 5c).

Fig. 6 presents noise removal results on a PD measurement performed for the PD experimental model due to surface discharge (Fig. 2c). It again demonstrates the applicability of the proposed method on removing the stochastic noise and extracting PD signals.

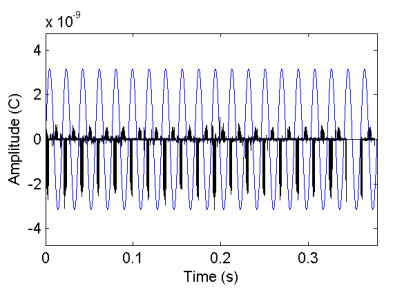

(a) Original signal

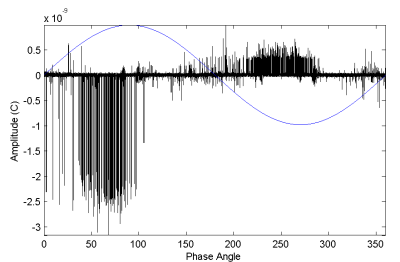

(c) Original phase-resolved partial discharge (PRPD)

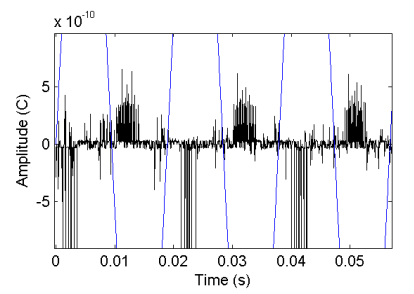

(b) Original signal (magnified)

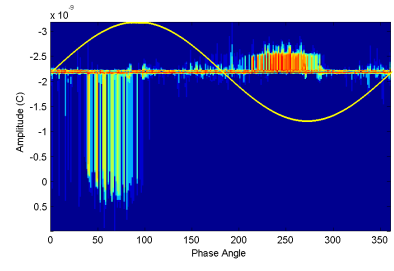

(d) Noise-removed phase-resolved partial discharge (PRPD)
Figure 6. Noise removal results of surface discharge.

In field PD measurements of transformers, multiple PD sources may always co-exist. Therefore, it is necessary to evaluate the performance of the proposed method on PD measurements of multiple PD sources. Fig. 7 presents noise removal results on multiple PD sources, which combine discharge with flat cavity and corona. Fig. 8 presents the results of another multiple PD sources, which combine discharge with flat cavity and surface discharge. It can be seen from both figures that the propose method can effectively remove stocahstic noise while PD signals generated by multiple PD sources can be retrieved.

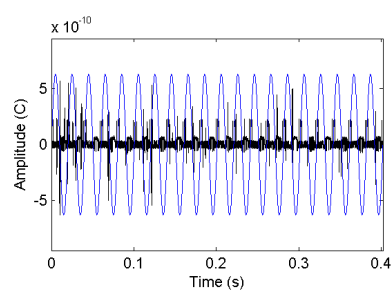

(a) Original signal

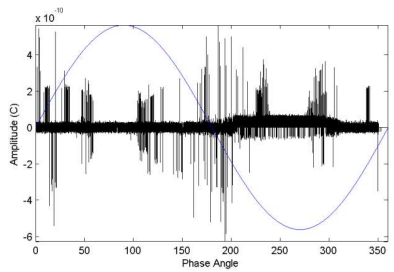

(c) Original phase-resolved partial discharge (PRPD)

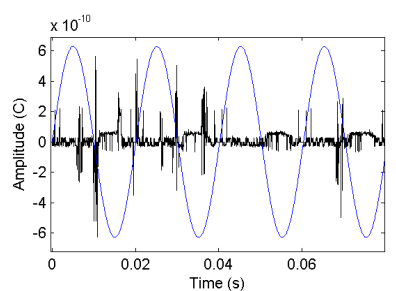

(b) Original signal (magnified)

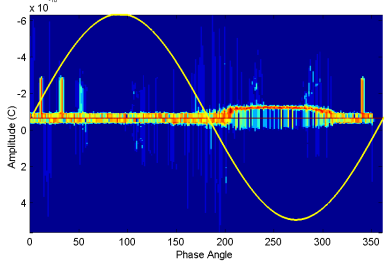

(d) Noise-removed phase-resolved partial discharge (PRPD)
Figure 7. Noise removal results of discharge with flat cavity and corona.

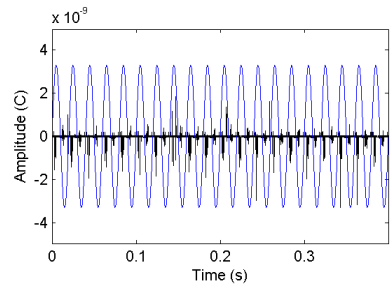

(a) Original signal

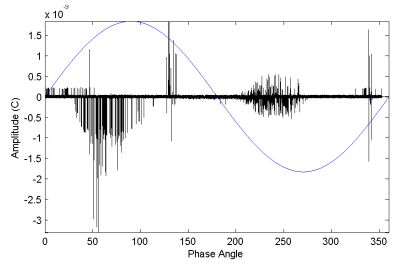

(c) Original phase-resolved partial discharge (PRPD)

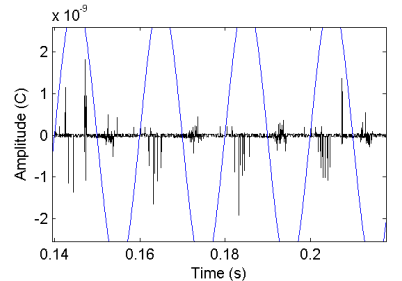

(b) Original signal (magnified)

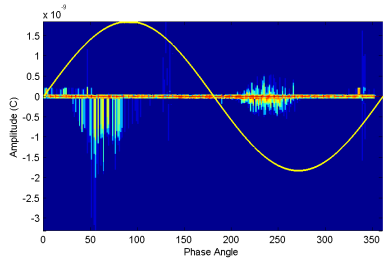

(d) Noise-removed phase-resolved partial discharge (PRPD)
Figure 8. Noise removal results of discharge with flat cavity and surface discharge.

\section{B. Noise Removal Evaluation on a Substation Transformer}

The proposed method is also applied to extract PD signals collected from a PD measurement on a 5 MVA power transformer with $22 \mathrm{kV}$ test voltage as shown in Fig. 9.

It can be seen that the original PD signal is immersed in noise and hardly to be identified (Fig. 9a to Fig. 9c). On the contrary, PD signal can be revealed by using the proposed method (Fig. 9d). The PD signal located near the negative cycle may be related to corona, while the PD signal located in both negative and positive cycles may be generated by internal discharge. 


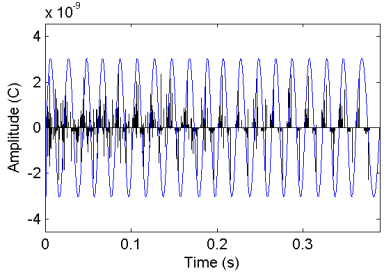

(a) Original signal

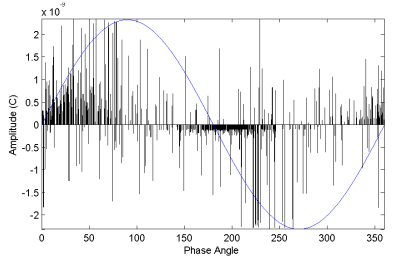

(c) Original phase-resolved partial discharge (PRPD)

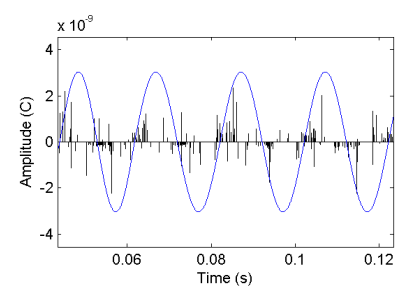

(b) Original signal (magnified)

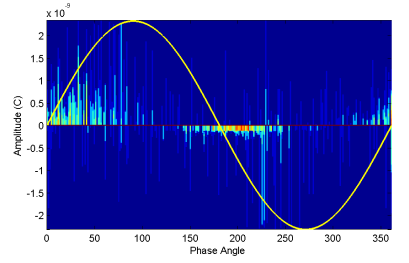
partial discharge (PRPD) (d) Noise-removed phase-resolved

Figure 9. Noise removal results of PD signals collected from a transformer.

\section{Comparison with a Wavelet Transform-Based Method}

This paper also compares performance of the proposed method with that of a discrete wavelet transform (DWT)based noise removal method. In DWT, the decomposed wavelet coefficients combine an approximation coefficient and detail coefficients. The approximation coefficient contains the lowest frequency signal component in an original signal while the detail coefficients contain the other frequency components in different frequency scales. The decomposition results of DWT using different wavelet functions for a PD signal collected from the experimental model on discharge with flat cavity (Fig. 2a) is shown in Fig. 10.

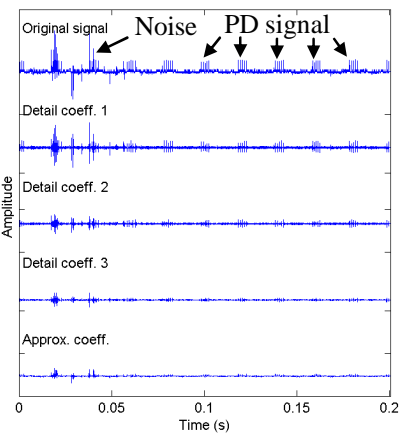

(a) Decomposition using db5

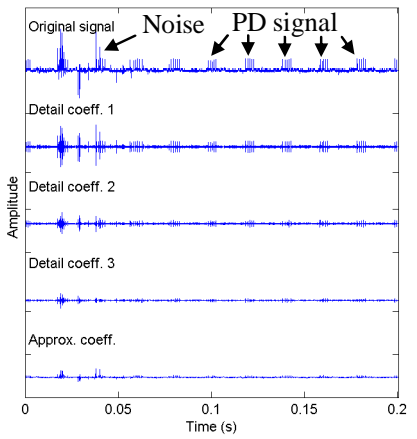

(b) Decomposition using bior 1.5
Figure 10. Discrete wavelet decomposition using different wavelet functions for a PD signal generated from discharge with flat cavity.

The original signal in Fig. 10 is the same as the signal presented in Fig. 4a. The decompositions are based on wavelet functions, $\mathrm{db} 5$ and bior1.5, which are commonly used in removing noise in PD measurements [1], [3], [6]. From the figure, it can be seen that PD signal cannot be separated from noise in the decomposed coefficients for both of the wavelet functions. Since the PD signal and the stochastic noise share the same frequency scale, they are located in the same coefficient after wavelet decomposition. Another reason for the noise cannot be separated from the PD signal is due to their similar pulses' shapes in which the wavelet functions cannot be able to distinguish them during decomposition process [3], [16]. The results indicate that wavelet transform-based noise removal method is not suitable for removing stochastic noise. On the contrary, the method proposed in this paper can effectively remove severe stochastic noise and extract PD signals.

\section{CONCLUSIONS}

This paper proposes a hybrid of fractal dimension and entropy to effectively remove stochastic noise and extract PD signals. This will be beneficial to transformer insulation diagnoses. Future works will be implemented for further verification of the proposed method by conducting more PD measurements on in-service transformers.

\section{ACKNOWLEDGMENT}

The authors gratefully acknowledge Australian Research Council, Powerlink Queensland, Energex, Ergon Energy, and TransGrid for providing supports for this work.

\section{REFERENCES}

[1] X. Song, C. Zhou, D. Hepburn, and G. Zhang, "Second generation wavelet transform for data denoising in PD measurement," IEEE Trans. Dielectr. Electr. Insul., vol. 14, pp. 1531-1537, 2007.

[2] E. Gulski, "Digital analysis of partial discharges," IEEE Trans. Dielectr. Electr. Insul., vol. 2, pp. 822-837, 1995.

[3] X. Ma, C. Zhou, and I. Kemp, "Automated wavelet selection and thresholding for PD detection," IEEE Elect. Insul. Mag., vol. 18, pp. 37-45, 2002.

[4] X. Zhou, C. Zhou, and I. Kemp, "An improved methodology for application of wavelet transform to partial discharge measurement denoising," IEEE Trans. Dielectr. Electr. Insul., vol. 12, pp. 586-594, 2005.

[5] X. Ma, C. Zhou, and I. Kemp, "Interpretation of wavelet analysis and its application in partial discharge detection," IEEE Trans. Dielectr. Electr. Insul., vol. 9, pp. 446-457, 2002.

[6] H. Zhang, T. Blackburn, B. Phung, and D. Sen, "A novel wavelet transform technique for on-line partial discharge measurements part 1: WT de-noising algorithm," IEEE Trans. Dielectr. Electr. Insul., vol. 14, pp. 3-14, 2007.

[7] S. Happe, H. Kranz, and W. Krause, "Advanced suppression of stochastic pulse shaped partial discharge disturbances," IEEE Trans. Dielectr. Electr. Insul., vol. 12, pp. 265-275, 2005.

[8] J. Chan, H. Ma, T. Saha, and C. Ekanayake, "A novel level-based automatic wavelet selection scheme for partial discharge measurement," Proc. Australasian Universities Power Eng. Conf., Bali, Indonesia, pp. 1-6, 2012.

[9] B. Mandelbrot, The Fractal Geometry of Nature, New York: Freeman, 1983.

[10] IEC International Standard 60270, "High-voltage test techniques - partial discharge measurements," 3rd edition, 2000.

[11] A. Balghonaim and J. Keller, "A maximum likelihood estimate for twovariable fractal surface," IEEE Trans. Image Process., vol. 7, pp. 1746$1753,1998$.

[12] J. Li, Q. Du, and C. Sun, "An improved box-counting method for image fractal dimension estimation," Pat. Recog., vol. 42, pp. 2460-2469, 2009.

[13] J. Li, T. Jiang, R. Harrison, and S. Grzybowski, "Recognition of ultra high frequency partial discharge signals using multi-scale features," IEEE Trans. Dielectr. Electr. Insul., vol. 19, pp. 1412-1420, 2012.

[14] F. Gu, H. Chang, and C. Kuo, "Gas-insulated switchgear PD signal analysis based on Hilbert-Huang transform with fractal parameters enhancement," IEEE Trans. Dielectr. Electr. Insul., vol. 20, pp. 1049-1055, 2013.

[15] G. Luo, D. Zhang, Y. Koh, K. Ng, and W. Leong, "Time-frequency entropy-based partial-discharge extraction for nonintrusive measurement," IEEE Trans. Power Del., vol. 27, pp. 1919-1927, 2012.

[16] A. Contin and S. Pastore, "Classification and separation of partial discharge signals by means of their auto-correlation function evaluation," IEEE Trans. Dielectr. Electr. Insul., vol. 16, pp. 1609-1622, 2009. 